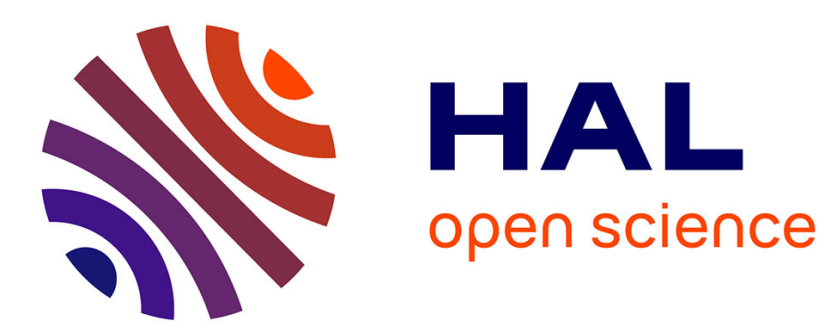

\title{
Influence of an interface domain wall on spin-valve giant magnetoresistance
}

\author{
Thomas Hauet, F Montaigne, M. Hehn, Y Henry, S Mangin
}

\section{To cite this version:}

Thomas Hauet, F Montaigne, M. Hehn, Y Henry, S Mangin. Influence of an interface domain wall on spin-valve giant magnetoresistance. Applied Physics Letters, 2008, 10.1063/1.3041640 . hal01345244

\section{HAL Id: hal-01345244 \\ https://hal.science/hal-01345244}

Submitted on 13 Jul 2016

HAL is a multi-disciplinary open access archive for the deposit and dissemination of scientific research documents, whether they are published or not. The documents may come from teaching and research institutions in France or abroad, or from public or private research centers.
L'archive ouverte pluridisciplinaire $\mathbf{H A L}$, est destinée au dépôt et à la diffusion de documents scientifiques de niveau recherche, publiés ou non, émanant des établissements d'enseignement et de recherche français ou étrangers, des laboratoires publics ou privés. 


\title{
Influence of an interface domain wall on spin-valve giant magnetoresistance
}

\author{
T. Hauet, ${ }^{1,2, a)}$ F. Montaigne, ${ }^{1}$ M. Hehn, ${ }^{1}$ Y. Henry, ${ }^{3}$ and S. Mangin ${ }^{1}$ \\ ${ }^{1}$ LPM, Nancy-University and CNRS, BP 239, F-54506 Vandoeuvre, France \\ ${ }^{2}$ Hitachi GST, Yerba Buena Rd 3403, San Jose, California 95135, USA \\ ${ }^{3}$ IPCMS, CNRS and Universite Louis Pasteur, BP 43, F-67037 Strasbourg Cedex 2, France
}

(Received 7 October 2008; accepted 12 November 2008; published online 4 December 2008)

\begin{abstract}
The magnetotransport properties of a $\mathrm{Gd}_{40} \mathrm{Fe}_{60} / \mathrm{Gd}_{10} \mathrm{Fe}_{90} / \mathrm{Cu} / \mathrm{Gd}_{40} \mathrm{Fe}_{60}$ spin-valve based on amorphous ferrimagnetic GdFe layers are reported. The $\mathrm{Gd}_{40} \mathrm{Fe}_{60} / \mathrm{Gd}_{10} \mathrm{Fe}_{90}$ bilayer is an exchange spring structure that allows an interfacial domain wall to be controlled by an applied field. As this domain wall is nucleated, compressed, and annihilated, changes in the spin-valve current-in-plane magnetoresistance are observed. After separating the various magnetoresistance contributions we could deduce the effect of the interface domain wall on both the giant and anisotropic magnetoresistances. () 2008 American Institute of Physics. [DOI: 10.1063/1.3041640]
\end{abstract}

Several architectures have been used to attain a better understanding of giant magnetoresistance (GMR) phenomenon. This important activity has been driven by its strong implications for magnetic data storage devices and sensors. ${ }^{1}$ In most GMR structures, the magnetic electrodes are sufficiently thin so that the magnetization could be considered as uniform across the thickness of the ferromagnetic layers. However, it is commonly known that GMR originates from a combination of interfacial and bulk spin-dependent scattering. Therefore, one may expect that a vertically nonuniform magnetization state of the magnetic layer would reflect in its GMR response. One way to induce a perpendicular-to-layer nonuniform magnetic configuration in a spin valve consists in replacing one of the magnetic electrodes with an exchange-coupled bilayer system. ${ }^{2-4}$ in which a planar interface domain wall (iDW) may be tuned.

In the present letter, we report on the magnetoresistance properties of such a modified spin-valve structure, $\mathrm{Gd}_{40} \mathrm{Fe}_{60} / \mathrm{Gd}_{10} \mathrm{Fe}_{90} / \mathrm{Cu} / \mathrm{Gd}_{40} \mathrm{Fe}_{60}$, where $\mathrm{Gd}_{x} \mathrm{Fe}_{1-x}$ are ferrimagnetic alloys. The alloys' magnetic properties (magnetization, anisotropy, exchange stiffness, etc.) can easily be tuned by changing composition ${ }^{5}$ and/or growth conditions. ${ }^{6}$ Such materials have already been used in spin-valve structures ${ }^{7,8}$ as well as in magnetic tunnel junctions. ' We focus on the magnetoresistance properties in a current-in-plane (CIP) geometry of a $\mathrm{Gd}_{40} \mathrm{Fe}_{60}(30 \mathrm{~nm}) / \mathrm{Gd}_{10} \mathrm{Fe}_{90}(30 \mathrm{~nm}) /$ $\mathrm{Cu}(6 \mathrm{~nm}) / \mathrm{Gd}_{40} \mathrm{Fe}_{60}(30 \mathrm{~nm})$ sample. The multilayer was grown from pure elements on a glass substrate kept at $77 \mathrm{~K}$ under high vacuum $\left(10^{-8}\right.$ Torr). In these conditions, both GdFe alloys are amorphous, and a uniaxial anisotropy axis is induced in the film plane. ${ }^{6}$ During the experiments described in the sequel, the external magnetic field is always applied along this anisotropy axis. In $\mathrm{Gd}_{x} \mathrm{Fe}_{1-x}$ the moments of the rare earth and those of the transition metal form two magnetic sublattices, which are coupled antiferromagnetically. In $\mathrm{Gd}_{40} \mathrm{Fe}_{60}$, the dominant sublattice magnetization is that of the rare earth whereas it is that of the transition metal for $\mathrm{Gd}_{10} \mathrm{Fe}_{90}$ [Fig. 1(a)]. With the exchange interaction across the interface being dominated by the ferromagnetic coupling between $\mathrm{Fe}$ spins, the net magnetization of the $\mathrm{Gd}_{40} \mathrm{Fe}_{60}$ and

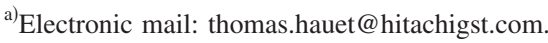

$\mathrm{Gd}_{10} \mathrm{Fe}_{90}$ layers are coupled antiferromagnetically [Fig. 1(a) and left sketch in Fig. 1(c)].

The spin valve was etched to obtain two patterned $100 \times 400 \mu \mathrm{m}^{2}$ stripes with the long axis either parallel or perpendicular to the well-defined easy axis of the GdFe layers [Fig. 1(b)]. Electrical contacts were added at the ends of the stripes by a gold lift-off process. Electrical measurements were performed using an ac excitation current of $1 \mathrm{~mA}$. The change in resistance as a function of field, $\Delta R(H)=R(H)$ $-R(H=0)$, was simultaneously monitored for the two stripes.
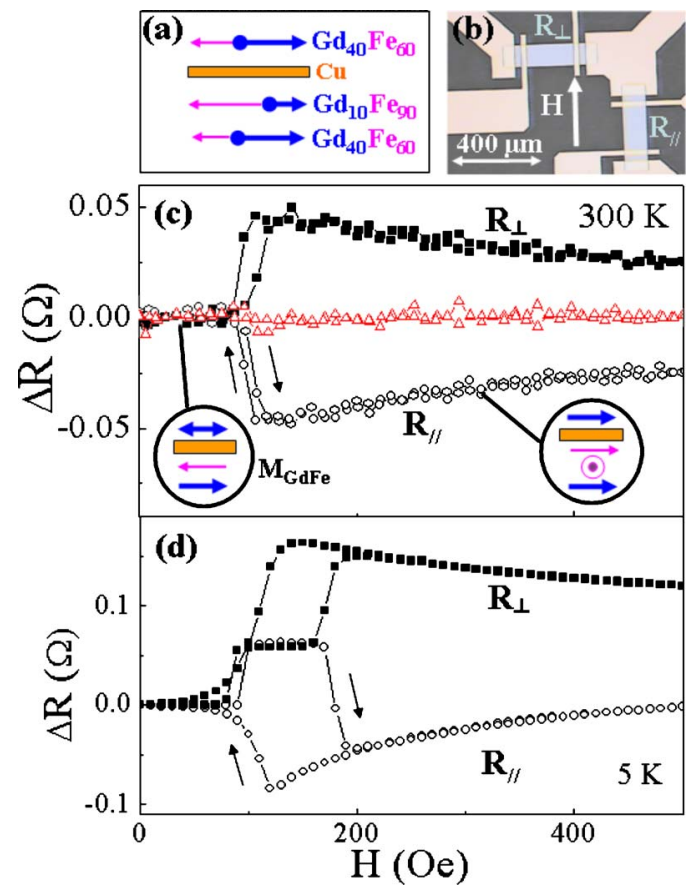

FIG. 1. (Color online) The sketch in (a) represents the Gd (thick blue arrows) and $\mathrm{Fe}$ (thin pink arrows) sublattices at 0 Oe after positive saturation. The scanning electron microscopy picture in (b) represents the two spinvalve wires (blue rectangles) used for CIP four-probe measurement. Relative magnetoresistance for the two wires with the current flowing parallel $\left(\Delta R_{\|}\right.$ open circle) and perpendicular ( $\Delta R_{\perp}$, full squares) to the magnetic field $(H)$ for 300 (c) and $5 \mathrm{~K}$ (d). The red open triangles in (c) correspond to the average of $\Delta R_{\|}$and $\Delta R_{\perp}$. The encircled sketches in (c) correspond to the average GdFe magnetization orientation with thick blue (thin pink) arrows when $\mathrm{Gd}(\mathrm{Fe})$ dominates. The encircled dot in the left sketch of (c) symbolizes an iDW. 
This procedure allows first removing anisotropic magnetoresistance (AMR) from the magnetoresistive signals by summing the magnetoresistance curves measured with the current lines parallel $\left(\Delta R_{\|}\right)$and perpendicular $\left(\Delta R_{\perp}\right)$ to the applied field. ${ }^{4,10}$ Moreover, subtracting those two signals removes any magnetoresistance contributions such as GMR that are independent of the in-plane current direction. ${ }^{4}$

Figure 1(c) shows the results of magnetotransport measurements performed on perpendicular stripes at $300 \mathrm{~K}$. For clarity reasons, magnetoresistive signals, measured after positive saturation, are shown for positive field only. The black arrows represent the field sweep direction. The sum of the magnetoresistances of the two stripes is zero indicating that only AMR effect is detectable here. The $300 \mathrm{~K}$ curves shown in Fig. 1(c) are indeed typical of the AMR signal in antiferromagnetically coupled bilayers ${ }^{3,4,10}$ and can be understood as described in the following for $\Delta R_{\|}(H)$. First, one has to note that the reversal of the single $\mathrm{Gd}_{40} \mathrm{Fe}_{60}$ layer is undetectable as far as AMR is concerned since the $\mathrm{Gd}_{40} \mathrm{Fe}_{60}$ magnetization is kept along its uniaxial anisotropy axis. As a consequence the AMR signal comes only from the $\mathrm{Gd}_{40} \mathrm{Fe}_{60}(30 \mathrm{~nm}) / \mathrm{Gd}_{10} \mathrm{Fe}_{90}(30 \mathrm{~nm})$ bilayer. In low fields, the magnetizations of the $\mathrm{Gd}_{40} \mathrm{Fe}_{60}$ and $\mathrm{Gd}_{10} \mathrm{Fe}_{90}$, inside the bilayer, are antiparallel to each other, along the anisotropy axis so that the AMR is minimum [Fig. 1(c)]. At $H \sim 100 \mathrm{Oe}$, because of the competition between the external field and the antiferromagnetic coupling at the interface, an iDW nucleates [Fig. 1(c)]. Inside the iDW the magnetic moments have a component perpendicular to the current direction so that the iDW nucleation gives rise to a negative jump in the AMR contribution. For $|H|>100 \mathrm{Oe}$, as the field increases, the iDW is compressed. The number of moments perpendicular to the current inside the iDW decreases and the AMR tends to reach back its maximum value. Note that no substantial GMR effect is measured at room temperature.

In Fig. 1(d), the $5 \mathrm{~K}$ magnetoresistances present a more complex behavior. In large applied fields, the curves of the two stripes show behavior similar to the one observed at $300 \mathrm{~K} . \Delta R_{\perp}$ slowly decreases as $\Delta R_{\|}$symmetrically increases. However, for low field, several jumps of resistance can be distinguished. To help us investigate the origin of the resistance variations $\Delta R_{\text {diff }}(H)=\frac{1}{2}\left[\Delta R_{\|}(H)-\Delta R_{\perp}(H)\right]$ and $\Delta R_{\text {sum }}=\frac{1}{2}\left[\Delta R_{\|}(H)+\Delta R_{\perp}(H)\right]$ are plotted in Figs. 2(a) and 2(b), respectively. $\Delta R_{\text {diff }}(H)$ is similar to the $\Delta R_{\|}$signal at $300 \mathrm{~K}$ and is again characteristic of the AMR signal recorded on antiferromagnetically exchange-coupled bilayers. ${ }^{3,4,10}$ To obtain quantitative details about the magnetic configurations, we compare the experimental curves with a micromagnetic model assuming a one-dimensional (1D) spin chain as described in Refs. 2, 3, and 11. In that calculation, the spin valve is treated as a chain of spins normal to the film plane where the two magnetic subsystems, the single $\mathrm{Gd}_{40} \mathrm{Fe}_{60}(30 \mathrm{~nm})$ layer on one hand and the $\mathrm{Gd}_{40} \mathrm{Fe}_{60}(30 \mathrm{~nm}) / \mathrm{Gd}_{10} \mathrm{Fe}_{90}(30 \mathrm{~nm})$ bilayer on the other hand, behave independently. Indeed no significant magnetic interlayer coupling through the 6-nm-thick $\mathrm{Cu}$ spacer layer is expected. The model uses a net magnetization of the ferrimagnetic materials, $M\left(\mathrm{Gd}_{40} \mathrm{Fe}_{60}\right)$ $=1000 \mathrm{emu} / \mathrm{cm}^{3}$ and $M\left(\mathrm{Gd}_{10} \mathrm{Fe}_{90}\right)=677 \mathrm{emu} / \mathrm{cm}^{3}$, anisotropy constants $K\left(\mathrm{Gd}_{40} \mathrm{Fe}_{60}\right)=10^{5} \mathrm{erg} / \mathrm{cm}^{3}$ and $K\left(\mathrm{Gd}_{10} \mathrm{Fe}_{90}\right)$ $=4 \times 10^{4} \mathrm{erg} / \mathrm{cm}^{3}$, bulk exchange stiffness $A\left(\mathrm{Gd}_{40} \mathrm{Fe}_{60}\right)=5$ $\times 10^{-7} \mathrm{erg} / \mathrm{cm}, A\left(\mathrm{Gd}_{10} \mathrm{Fe}_{90}\right)=1 \times 10^{-7} \mathrm{erg} / \mathrm{cm}$, and an inter-

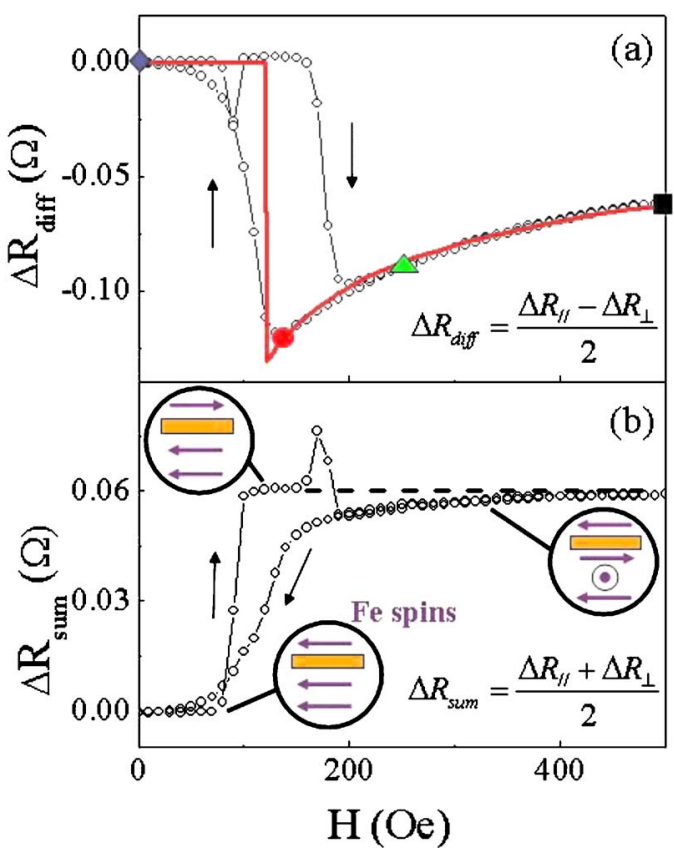

FIG. 2. (Color online) Magnetoresistance (open circle) vs magnetic field $(H)$ obtained after subtracting (a) and summing (b) the resistances of the two stripes. The red solid line in (a) is the AMR variation predicted from a $1 D$ model. The big symbols indicate the AMR values corresponding to the magnetic configuration shown in Fig. 3. In (b), the encircled sketches correspond to the $\mathrm{Fe}$ spin configuration and the dashed line is a guide for the eyes.

facial exchange coupling across the $\mathrm{Gd}_{40} \mathrm{Fe}_{60} / \mathrm{Gd}_{10} \mathrm{Fe}_{90}$ interface $J=1 \mathrm{erg} / \mathrm{cm}^{2}$ in accord with previous studies on single films and multilayers. ${ }^{3,5,10}$ The magnetic configuration corresponding to a minimum of the total magnetic energy is then deduced for each field. From this magnetic profile given by the magnetization angle depth dependence $\theta(z)$, the AMR signal is calculated as described in detail in Refs. 12 and 3. The calculated AMR [red solid line in Fig. 2(a)] is in good agreement with the extracted $\Delta R_{\text {diff }}$ results.

Contrary to the room temperature results [Fig. 1(c)], a residual contribution to the magnetoresistance $\left(\Delta R_{\text {sum }}\right)$ exists after AMR removal at $5 \mathrm{~K}$ [Fig. 2(b)]. $\Delta R_{\text {sum }}$ shows two well-defined resistance levels [Fig. 2(b)] that are observed neither for the single $\mathrm{GdFe}$ layer nor for the $\mathrm{Gd}_{40} \mathrm{Fe}_{60} / \mathrm{Gd}_{10} \mathrm{Fe}_{90}$ bilayers. ${ }^{10} \Delta R_{\text {sum }}(H)$ variations can be explained in terms of GMR effect arising from the $\mathrm{Gd}_{40} \mathrm{Fe}_{60} / \mathrm{Gd}_{10} \mathrm{Fe}_{90} / \mathrm{Cu} / \mathrm{Gd}_{40} \mathrm{Fe}_{60}$ spin valve. What is first striking is that the electrical resistance is maximum when the average GdFe magnetization vectors of the layers adjacent to the spacer are parallel to each other (for $80 \mathrm{Oe}<|H|$ $<180$ Oe, topmost plateaus), and minimum when they are antiparallel (for $|H|<80 \mathrm{Oe}$, bottommost plateau). Such an inverse GMR has already been reported for spin-valve structures containing rare-earth-transition metal alloys. ${ }^{7,8}$ In such systems, the GMR effect is mainly governed by the spindependent scattering on $\mathrm{Fe}$ atoms, which is comparatively much stronger than on rare-earth atoms. Hence, one should only focus on how the orientation of the Fe spin subnetwork varies with field as depicted by the sketches in Fig. 2(b). The high (low) resistance level is found for an antiparallel (parallel) alignment of the Fe moments on each side of the copper spacer. 


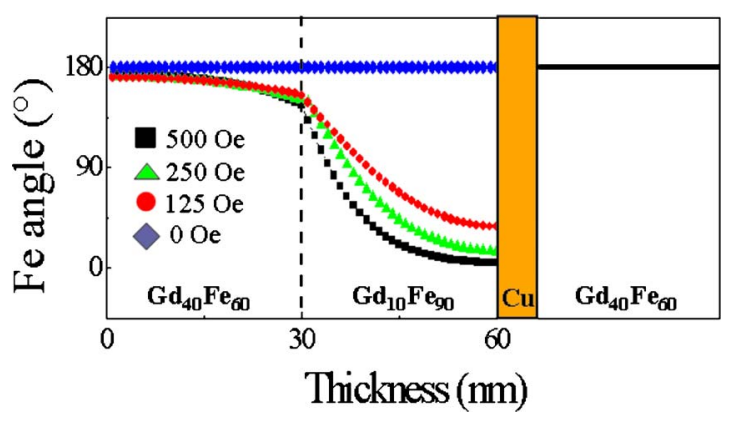

FIG. 3. (Color online) Theoretical variation of the azimuthal angle of the Fe sublattice magnetization as a function of depth, for decreasing values of the applied magnetic field. The zero angle corresponds to the positive field direction.

Intermediate GMR values between the high and low resistance levels are measured when an iDW lies in the $\mathrm{Gd}_{40} \mathrm{Fe}_{60} / \mathrm{Gd}_{10} \mathrm{Fe}_{90}$ bilayer [Fig. 2(b)]. Interestingly, $\Delta R_{\text {sum }}$ saturates around $500 \mathrm{Oe}$ whereas $\Delta R_{\text {diff }}$ still indicates the presence of an iDW. This result is explained if the GMR is sensitive to the $\mathrm{Fe}$ moments present at both $\mathrm{Cu}$ interfaces only. Within this assumption, GMR saturation is reached when the magnetization inside the $\mathrm{Gd}_{10} \mathrm{Fe}_{90}$ is uniform but also when the iDW is sufficiently thin, i.e., for large applied fields, so that the Fe moments at the copper interface are antiparallel to the $\mathrm{Fe}$ spins inside the single $\mathrm{Gd}_{40} \mathrm{Fe}_{60}$ layer. Indeed, for $125 \mathrm{Oe}<H<500 \mathrm{Oe}$, the micromagnetic calculation shows that iDW is not a "180" domain wall (Fig. 3). The Fe moments at the $\mathrm{Gd}_{10} \mathrm{Fe}_{90} / \mathrm{Cu}$ interface are found to be slightly titled from the anisotropy axis. As the field increases the $\mathrm{Fe}$ spins in the vicinity of the $\mathrm{Gd}_{10} \mathrm{Fe}_{90} / \mathrm{Cu}$ interface rotate progressively, which can be correlated to the continuous evolution of the intermediate GMR values [Fig. 2(b) $]{ }^{13}$ Close to $500 \mathrm{Oe}$, in accordance with the micromagnetic calculation, the $\mathrm{Fe}$ spins at the $\mathrm{Gd}_{10} \mathrm{Fe}_{90} / \mathrm{Cu}$ interface become antiparallel to the $\mathrm{Fe}$ spins inside the single $\mathrm{Gd}_{40} \mathrm{Fe}_{60}$ layer (Fig. 3). Consequently, the GMR signal saturates [Fig. 2(b)] above 500 Oe. The reason why the GMR signal depends on the $\mathrm{Fe}$ moments' orientation within a very thin depth at the $\mathrm{Cu}$ interface only is to be found in the very short mean free path in the magnetic alloys used. The mean free path of amorphous rare-earth-transition metal alloys is expected to be of the order of $1 \mathrm{~nm}$ at most. In the CIP geometry, it is usually admitted that only a limited fraction of the magnetic layers of thickness of the order of the mean free path next to the interfaces with the spacer layer contributes to GMR. ${ }^{14}$ As a consequence, in $\mathrm{Gd}_{40} \mathrm{Fe}_{60} / \mathrm{Gd}_{10} \mathrm{Fe}_{90} / \mathrm{Cu} /$ $\mathrm{Gd}_{40} \mathrm{Fe}_{60}$, whereas changes in AMR reflect the iDW (de)compression process in the entire $\mathrm{Gd}_{40} \mathrm{Fe}_{60} / \mathrm{Gd}_{10} \mathrm{Fe}_{90}$ bilayer, GMR changes arise only from the progressive rotation of the $\mathrm{Gd}_{10} \mathrm{Fe}_{90}$ magnetization in the vicinity of the interface with copper (Fig. 3).

The present work demonstrates that a spin-valve structure including a domain wall at the interface with the spacer layer, e.g., via a spring magnet, provides a new way to study quantitatively spin-dependent transport lengths (or conversely to probe the magnetic configurations). Indeed for polycrystalline or crystalline magnetic materials that can accept DW thickness comparable to electron spin diffusion length, a large part of the spins in the domain wall should contribute to the GMR effect. In the view of quantitative results, the sensitivity of this method should be improved by tuning the thickness of the studied magnetic layer, i.e., containing the main part of the iDW (in our case $\mathrm{Gd}_{10} \mathrm{Fe}_{90}$ ). An advantage of the present approach is that results can be obtained on one single sample and does not depend on the sample-sample reproducibility as other techniques reported in literature. ${ }^{8,15}$

In conclusion, the effect of an iDW on the magnetoresistive properties of a spin-valve $\mathrm{Gd}_{40} \mathrm{Fe}_{60} / \mathrm{Gd}_{10} \mathrm{Fe}_{90} / \mathrm{Cu} /$ $\mathrm{Gd}_{40} \mathrm{Fe}_{60}$ has been studied. Simultaneous CIP resistivity measurements on two perpendicular wires allowed separating AMR and GMR signals. The AMR variations are found to arise from the entire depth magnetic configuration over the $\mathrm{Gd}_{40} \mathrm{Fe}_{60} / \mathrm{Gd}_{10} \mathrm{Fe}_{90}$ bilayer. On the contrary, the GMR effect only depends on the relative iron moments' orientation at the $\mathrm{GdFe} / \mathrm{Cu}$ interfaces due to the low mean free path of the amorphous GdFe layer. Our work defines a new method to study the magnetotransport characteristic lengths, which can be applied at spintronic structures such as spin valves or magnetic tunnel junctions.

\footnotetext{
${ }^{1}$ Magnetic Multilayers and Giant Magnetoresistance: Fundamentals and Industrial Applications, edited by B. U. Hartmann (Springer, Berlin, 2000).

${ }^{2}$ T. Hauet, J. A. Borchers, Ph. Mangin, Y. Henry, and S. Mangin, Phys. Rev. Lett. 96, 067207 (2006).

${ }^{3}$ Y. Henry, S. Mangin, T. Hauet, and F. Montaigne, Phys. Rev. B 73 , 134420 (2006); F. Canet, S. Mangin, C. Bellouard, and M. Piecuch, Europhys. Lett. 52, 594 (2000).

${ }^{4}$ S. N. Gordeev, J.-M. L. Beaujour, G. J. Bowden, B. D. Rainford, P. A. J. de Groot, R. C. C. Ward, M. R. Wells, and A. G. M. Jansen, Phys. Rev. Lett. 87, 186808 (2001) and references therein.

${ }^{5}$ P. Hansen, C. Clausen, G. Much, M. Rosenkranz, and K. Witter, J. Appl. Phys. 66, 756 (1989).

${ }^{6}$ S. Mangin, C. Bellouard, G. Marchal, and B. Barbara, J. Magn. Magn. Mater. 165, 161 (1997).

${ }^{7}$ C. Bellouard, H. D. Rapp, B. George, S. Mangin, G. Marchal, and J. C. Ousset, Phys. Rev. B 53, 5082 (1996).

${ }^{8}$ D. Z. Yang, B. You, X. X. Zhang, T. R. Gao, S. M. Zhou, and J. Du, Phys. Rev. B 74, 024411 (2006).

${ }^{9}$ C. Kaiser and S. S. P. Parkin, Appl. Phys. Lett. 88, 112511 (2006).

${ }^{10}$ T. Hauet, F. Montaigne, M. Hehn, Y. Henry, and S. Mangin, Phys. Rev. B (submitted).

${ }^{11}$ E. E. Fullerton, J. S. Jiang, M. Grimsditch, C. H. Sowers, and S. D. Bader, Phys. Rev. B 58, 12193 (1998).

${ }^{12}$ F. Montaigne, S. Mangin, and Y. Henry, Phys. Rev. B 67, 144412 (2003).

${ }^{13}$ Note that intermediate values of $\Delta R_{\text {sum }}(H)$ that are obtained for $75 \mathrm{Oe}$ $<H<125$ Oe arise certainly because of the field distribution in the GdFe magnetization reversal that can already be seen in the $\Delta R_{\|}$and $\Delta R_{\perp}$ measurements shown in Fig. 1. In that case intermediate GMR values would be caused by the formation of lateral domain.

${ }^{14}$ S. Y. Hsu, A. Barthelemy, P. Holody, L. Loloee, P. A. Schroeder, and A. Fert, Phys. Rev. Lett. 78, 2652 (1997).

${ }^{15}$ S. S. P. Parkin, Phys. Rev. Lett. 71, 1641 (1993).
} 